\title{
Universal Current-Mode Biquad Filter Using a VDTA
}

\author{
Dinesh Prasad $^{1 *}$, Data Ram Bhaskar ${ }^{1}$, Mayank Srivastava ${ }^{2}$ \\ ${ }^{1}$ Department of Electronics and Communication Engineering, Faculty of Engineering and Technology, \\ Jamia Millia Islamia, New Delhi, India \\ ${ }^{2}$ D/O Electronics and Communication Engineering, Amity School of Engineering and Technology, \\ Amity University, Noida, India \\ Email: *dprasad@jmi.ac.in,dbhaskar@jmi.ac.in, mayank2780@gmail.com
}

Received October 9, 2012; revised November 9, 2012; accepted November 16, 2012

\begin{abstract}
This paper presents a new current-mode single input multi output (SIMO) type biquad employing one voltage differencing transconductance amplifier (VDTA), two grounded capacitors and a single grounded resistor. The configuration realizes all basic filter functions (i.e. Low Pass (LP), High Pass (HP), Band Pass (BP), Notch (BR) and All Pass (AP)). The natural frequency $\left(\omega_{0}\right)$ and bandwidth $(\mathrm{BW})$ are independently tunable. The workability of proposed configuration has been verified using SPICE simulation with TSMC CMOS $0.18 \mu \mathrm{m}$ process parameters.
\end{abstract}

Keywords: Current Mode Filter; Voltage Differencing Transconductance Amplifier

\section{Introduction}

Active filters are important basic building blocks, which are frequently employed in electrical engineering applications. The current-mode approach [1] in designing active filters has become more popular due to its advantageous features such as larger dynamic range and wider bandwidth as compared to voltage-mode counterparts (particularly for the high frequency operations), simpler filtering configurations and lower power consumption. During the past few years, active filters using different current-mode/voltage-mode building blocks have been employed in which VDTA, recently introduced in [2], appears to be a useful active building block for an easy CMOS implementation of current-mode signal processing/signal generation [3-5].

Various SIMO-type active filters using different active elements are available in the literature see [6-13] and the references cited therein. In references [6-9], SIMO-type filter configurations employing $2 / 4$ resistors and $2 / 3$ capacitors have been presented. These proposed filter structures do not realize all the five filter responses. The configurations presented in [10] and [11] although use one resistor and two capacitors but fail to realize all the basic filter functions. Although the biquads proposed in [12] and [13] realize all the five filter functions but they use two capacitors along with $2 / 3$ resistors. Therefore, the purpose of this communication is to propose a new SIMO-type current-mode universal biquad filter employing one VDTA, two grounded capacitors and a sin-

*Corresponding author. gle grounded resistor, which realizes all the basic filter functions i.e. LP, HP, BP, BR and AP. The natural frequency $\omega_{0}$ and $\mathrm{BW}$ are independently tunable. The proposed circuit also offers low active and passive sensitivities. The workability of proposed configuration has been verified using SPICE simulation with TSMC CMOS 0.18 $\mu \mathrm{m}$ process parameters.

\section{The Proposed New Configurations}

The symbolic notation of the VDTA is shown in Figure 1, where $V_{P}$ and $V_{N}$ are input terminals and $Z, X^{+}$and $X^{-}$ are output terminals. All terminals of VDTA exhibit high impedance values [2]. The VDTA can be described by the following set of equations:

$$
\left[\begin{array}{c}
I_{Z} \\
I_{X^{+}} \\
I_{X^{-}}
\end{array}\right]=\left[\begin{array}{ccc}
g_{m_{1}} & -g_{m_{1}} & 0 \\
0 & 0 & g_{m_{2}} \\
0 & 0 & -g_{m_{2}}
\end{array}\right]\left[\begin{array}{c}
V_{V_{P}} \\
V_{V_{N}} \\
V_{Z}
\end{array}\right]
$$

2.

The proposed circuit configuration is shown in Figure

A routine circuit analysis of Figure 2 yields the following filter transfer functions:

$$
\begin{gathered}
\left.T_{1}(s)\right|_{L P}=\frac{I_{o 1}}{I_{\text {in }}}=\frac{g_{m_{1}} g_{m_{2}}}{D(s)} \\
\left.T_{2}(s)\right|_{B P}=\frac{I_{o 2}}{I_{\text {in }}}=-\frac{s\left(\frac{g_{m_{1}}}{C_{1}}\right)}{D(s)}
\end{gathered}
$$




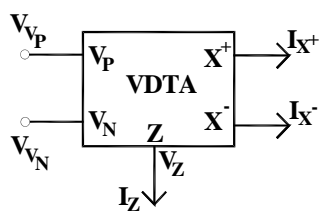

Figure 1. The symbolic notation of VDTA.

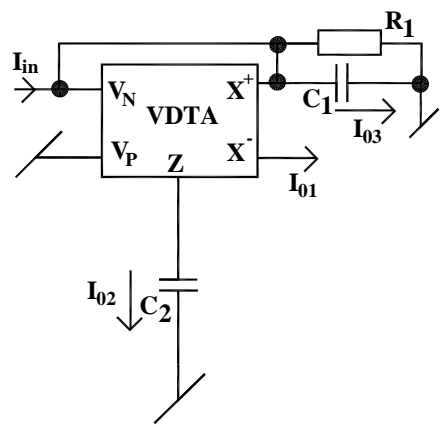

Figure 2. The proposed configuration.

$$
\begin{gathered}
\left.T_{3}(s)\right|_{H P}=\frac{I_{o 3}}{I_{i n}}=\frac{s^{2}}{D(s)} \\
\left.T_{4}(s)\right|_{\text {NOTCH }}=\frac{\left(I_{o 3}+I_{o 1}\right)}{I_{\text {in }}}=\frac{\left(s^{2}+\frac{g_{m_{1}} g_{m_{2}}}{C_{1} C_{2}}\right)}{D(s)} \\
\left.T_{5}(s)\right|_{A P}=\frac{\left(I_{o 3}+I_{o 2}+I_{o 1}\right)}{I_{\text {in }}}=\frac{\left\{s^{2}-s\left(\frac{g_{m_{1}}}{C_{1}}\right)+\frac{g_{m_{1}} g_{m_{2}}}{C_{1} C_{2}}\right\}}{D(s)}
\end{gathered}
$$

where

$$
D(s)=s^{2}+s\left(\frac{1}{R_{1} C_{1}}\right)+\frac{g_{m_{1}} g_{m_{2}}}{C_{1} C_{2}}
$$

The natural frequency $\omega_{0}$, BW and quality factor $Q_{0}$ are given by:

$$
\begin{gathered}
\omega_{0}=\sqrt{\frac{g_{m_{1}} g_{m_{2}}}{C_{1} C_{2}}} \\
B W=\frac{1}{R_{1} C_{1}} \\
Q_{0}=\sqrt{\frac{g_{m_{1}} g_{m_{2}} C_{1} R_{1}^{2}}{C_{2}}}
\end{gathered}
$$

\section{SPICE Simulation Results}

To verify the theoretical analysis, the proposed configuration was simulated using CMOS VDTA from [2]. Power supply voltages were taken as $V_{D D}=-V_{S S}=1 \mathrm{~V}$ and $I_{B 1}=I_{B 2}=150 \mu \mathrm{A}, I_{B 3}=I_{B 4}=42.38 \mu \mathrm{A}$ biasing currents are used. The passive elements of the configuration were selected as $C_{1}=C_{2}=0.01 \mathrm{nF}$ and $R_{1}=1.58 \mathrm{~K} \Omega$. The transconductances of VDTA were controlled by bias currents. Figure 3 shows the simulated filter responses of LP, BP, HP, BR and AP. These results, thus, confirm the validity of the proposed configuration. A comparison with other SIMO-type current-mode biquads using a single active device is presented in Table 1.

\section{Conclusion}

A new current-mode SIMO-type biquad filter has been presented which uses only one VDTA and three grounded passive elements. The proposed filter can realize the second-order LP, BP, HP, BR and AP responses. The circuit employs all grounded passive components (which is desirable for IC implementation) and offers low active and passive sensitivities. The natural frequency $\left(\omega_{0}\right)$ and bandwidth (BW) are independently tunable. SPICE simulations have established the workability of the proposed formulation.

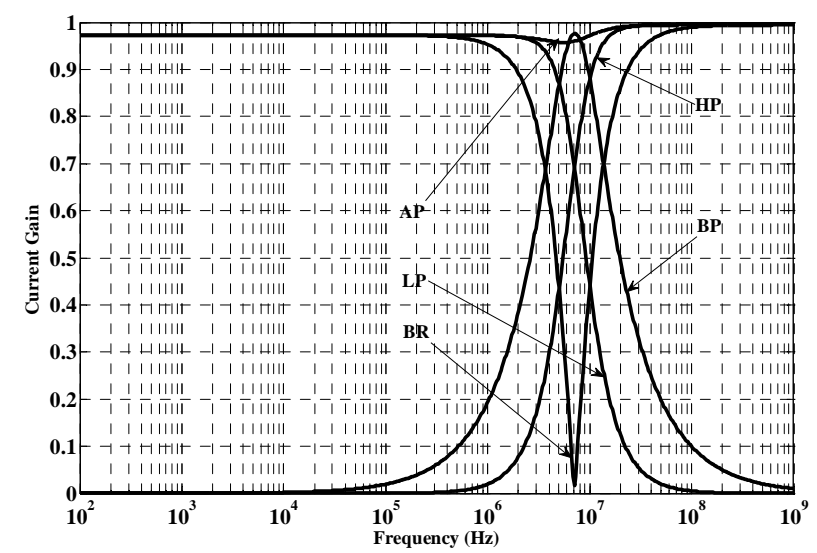

Figure 3. Frequency response.

Table 1. Comparison with other SIMO-type current-mode biquads using a single active device.

\begin{tabular}{ccccc}
\hline Reference & $\begin{array}{c}\text { No. of active } \\
\text { component }\end{array}$ & $\begin{array}{c}\text { No. of } \\
\text { resistors }\end{array}$ & $\begin{array}{c}\text { No. of } \\
\text { capacitors }\end{array}$ & $\begin{array}{c}\text { All five filter } \\
\text { function } \\
\text { realized }\end{array}$ \\
\hline$[6]$ & 1 & 4 & 2 & NO \\
{$[7]$} & 1 & $3 / 4$ & 3 & NO \\
{$[8]$} & 1 & 2 & 2 & NO \\
{$[9]$} & 1 & 2 & 2 & NO \\
{$[10]$} & 1 & 1 & 2 & NO \\
{$[11]$} & 1 & 1 & 2 & NO \\
{$[12]$} & 1 & 3 & 2 & YES \\
{$[13]$} & 1 & 2 & 2 & YES \\
Proposed & $\mathbf{1}$ & $\mathbf{1}$ & $\mathbf{2}$ & YES \\
\hline
\end{tabular}




\section{Acknowledgements}

This work was performed at the Advanced Analog Signal Processing Laboratory of the Department of Electronics and Communication Engineering, F/o Engineering and Technology, Jamia Millia Islamia, Jamia Nagar, New Delhi-110025, India.

\section{REFERENCES}

[1] C. Toumazau, F. J. Lidgey and D. G. Haigh, "Analogue IC Design: The Current-Mode Approach,” Peter Peregrinus Limited, London, 1990.

[2] D. Biolek, R. Senani, V. Biolkova and Z. Kolka, "Active Elements for Analog Signal Processing, Classification, Review and New Proposals," Radioengineering, Vol. 17, No. 4, 2008, pp. 15-32.

[3] A. Yesil, F. Kacar and H. Kuntman, "New Simple CMOS Realization of Voltage Differencing Transconductance Amplifier and Its RF Filter Application," Radioengineering, Vol. 20, No. 3, 2011, pp. 632-637.

[4] D. Prasad and D. R. Bhaskar, "Electronically-Controllable Explicit Current Output Sinusoidal Oscillator Employing Single VDTA," ISRN Electronics, Vol. 2012, 2012, Article ID: 382560 . doi: $10.5402 / 2012 / 382560$

[5] J. Satansup, T. Pukkalanun and W. Tangsrirat, "Electronically Tunable Single-Input Five-Output Voltage-Mode Universal Filter Using VDTAs and Grounded Passive Elements," Circuits, Systems, and Signal Processing, 2012 (online). doi:10.1007/s00034-012-9492-0

[6] A. Fabre and J. L. Houle, "Voltage-Mode and CurrentMode Sallen-Key Implementations Based on Translinear Conveyors," IEEE Proceedings-G: Circuits, Devices and
Systems, Vol.139, No.7, 1992, pp. 491-497.

[7] C. M. Chang, C. C. Chien and H. Y. Wang, "Universal Active Current Filters Using Single Second Generation Current Conveyor," Electronics Letters, Vol. 29, No. 13, 1993, pp. 1159-1160. doi:10.1049/el:19930775

[8] E. Yuce, B. Metin and O. Cicekoglu, "Current-Mode Biquadratic Filters Using Single CCIII and Minimum Number of Passive Elements," Frequenz: Journal of RF-Engineering and Telecommunications, Vol. 58, No. 9-10, 2004, pp. 225-227.

[9] B. Chaturvedi and S. Maheshwari, "Current Mode Biquad Filter with Minimum Component Count," Journal of Active and Passive Electronic Components, Vol. 2011, 2011, Article ID: 391642. doi:10.1155/2011/391642

[10] D. Biolek, V. Biolkova and Z. Kolka, "Current-Mode Biquad Employing Single CDTA," Indian Journla of Pure and Applied Physics, Vol. 47, No. 7, 2009, pp. 535537.

[11] W. Tangsrirat, "Novel Current-Mode and Voltage-Mode Universal Biquad Filters Using Single CFTA," Indian Journal of Engineering and Material Science, Vol. 17, 2010, pp. 99-104.

[12] C. N. Lee and C. M. Chang, "Single FDCCII-Based Mixed-Mode Biquad Filter with Eight Outputs," AEU: International Journal of Electronics and Communications, Vol. 63, No. 9, 2009, pp. 736-742. doi:10.1016/j.aeue.2008.06.015

[13] D. Prasad, D. R. Bhaskar and A. K. Singh, "Universal Current-Mode Biquad Filter Using Dual Output Current Differencing Transconductance Amplifier," AEU: International Journal of Electronics and Communications, Vol. 63, No. 6, 2009, pp. 497-501. doi:10.1016/j.aeue.2008.02.012 


\section{Appendix: Non-Ideal Analysis}

Considering the non-ideal effect of various parameters of VDTA i.e., the finite $X$-terminal parasitic impedance consisting of a resistance $R_{X}$ in parallel with capacitance $C_{X}$, the parasitic impedance at the $Z$-terminal consisting of a resistance $R_{Z}$ in parallel with capacitance $C_{Z}$, the parasitic impedance at the $V_{p}$-terminal consisting of a resistance $R_{p}$ in parallel with capacitance $C_{p}$ and the parasitic impedance at the $V_{n}$-terminal consisting of a resistance $R_{n}$ in parallel with capacitance $C_{n}$. The parasitic impedances belong to the circuit shown in Figure 2 are indicated in Figure A1.

Considering the above parasitic impedances, the natural frequency $\omega_{0}$ and quality factor $Q_{0}$ are found to be:

$$
\omega_{0}=\sqrt{\frac{\left(\frac{1}{R_{1}}+\frac{1}{R_{x}}+\frac{1}{R_{n}}\right) \frac{1}{R_{z}}+g_{m_{1}} g_{m_{2}}}{\left(C_{1} C_{2}+C_{1} C_{z}+C_{2} C_{x}+C_{z} C_{x}+C_{2} C_{n}+C_{n} C_{z}\right)}}
$$

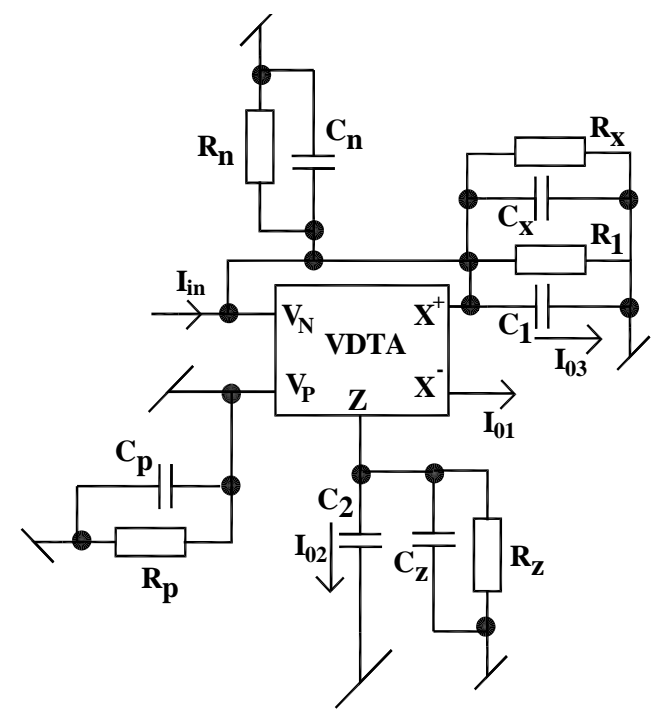

Figure A1. Parasitic impedances of VDTA affecting the circuit of Figure 2.

$$
Q_{0}=\frac{\sqrt{\left\{\left(\frac{1}{R_{1}}+\frac{1}{R_{x}}+\frac{1}{R_{n}}\right) \frac{1}{R_{z}}+g_{m_{1}} g_{m_{2}}\right\} *\left(C_{1} C_{2}+C_{1} C_{z}+C_{2} C_{x}+C_{z} C_{x}+C_{2} C_{n}+C_{n} C_{z}\right)}}{\left(\frac{C_{1}}{R_{z}}+\frac{C_{x}}{R_{z}}+\frac{C_{n}}{R_{z}}+\frac{C_{2}}{R_{1}}+\frac{C_{z}}{R_{1}}+\frac{C_{2}}{R_{x}}+\frac{C_{z}}{R_{x}}+\frac{C_{2}}{R_{n}}+\frac{C_{z}}{R_{n}}\right)}
$$

The sensitivity of $\omega_{0}$ and quality factor $Q_{0}$ with respect to its active and passive elements are given as:

$$
\begin{aligned}
& S_{g_{m_{1}}}^{\omega_{0}}=S_{g_{m_{2}}}^{\omega_{0}}=\frac{g_{m_{1}} g_{m_{2}}}{2\left\{\left(\frac{1}{R_{1}}+\frac{1}{R_{x}}+\frac{1}{R_{n}}\right) \frac{1}{R_{z}}+g_{m_{1}} g_{m_{2}}\right\}}, S_{R_{1}}^{\omega_{0}}=-\frac{\frac{1}{R_{1} R_{z}}}{2\left\{\left(\frac{1}{R_{1}}+\frac{1}{R_{x}}+\frac{1}{R_{n}}\right) \frac{1}{R_{z}}+g_{m_{1}} g_{m_{2}}\right\}} \\
& S_{R_{x}}^{\omega_{0}}=-\frac{\frac{1}{R_{x} R_{z}}}{2\left\{\left(\frac{1}{R_{1}}+\frac{1}{R_{x}}+\frac{1}{R_{n}}\right) \frac{1}{R_{z}}+g_{m_{1}} g_{m_{2}}\right\}}, S_{R_{n}}^{\omega_{0}}=-\frac{\frac{1}{R_{n} R_{z}}}{2\left\{\left(\frac{1}{R_{1}}+\frac{1}{R_{x}}+\frac{1}{R_{n}}\right) \frac{1}{R_{z}}+g_{m_{1}} g_{m_{2}}\right\}} \\
& S_{R_{z}}^{\omega_{0}}=-\frac{\frac{1}{R_{z}}\left(\frac{1}{R_{1}}+\frac{1}{R_{x}}+\frac{1}{R_{n}}\right)}{2\left\{\left(\frac{1}{R_{1}}+\frac{1}{R_{x}}+\frac{1}{R_{n}}\right) \frac{1}{R_{z}}+g_{m_{1}} g_{m_{2}}\right\}}, S_{C_{1}}^{\omega_{0}}=-\frac{C_{1}\left(C_{2}+C_{z}\right)}{2\left(C_{1} C_{2}+C_{1} C_{z}+C_{2} C_{x}+C_{z} C_{x}+C_{2} C_{n}+C_{n} C_{z}\right)} \\
& S_{C_{2}}^{\omega_{0}}=-\frac{C_{2}\left(C_{1}+C_{x}+C_{n}\right)}{2\left(C_{1} C_{2}+C_{1} C_{z}+C_{2} C_{x}+C_{z} C_{x}+C_{2} C_{n}+C_{n} C_{z}\right)}, S_{C_{z}}^{\omega_{0}}=-\frac{C_{z}\left(C_{1}+C_{x}+C_{n}\right)}{2\left(C_{1} C_{2}+C_{1} C_{z}+C_{2} C_{x}+C_{z} C_{x}+C_{2} C_{n}+C_{n} C_{z}\right)} \\
& S_{C_{x}}^{\omega_{0}}=-\frac{C_{x}\left(C_{2}+C_{z}\right)}{2\left(C_{1} C_{2}+C_{1} C_{z}+C_{2} C_{x}+C_{z} C_{x}+C_{2} C_{n}+C_{n} C_{z}\right)}, S_{C_{n}}^{\omega_{0}}=-\frac{C_{n}\left(C_{2}+C_{z}\right)}{2\left(C_{1} C_{2}+C_{1} C_{z}+C_{2} C_{x}+C_{z} C_{x}+C_{2} C_{n}+C_{n} C_{z}\right)} \\
& S_{g_{m_{1}}}^{Q_{0}}=S_{g_{m_{2}}}^{Q_{0}}=\frac{g_{m_{1}} g_{m_{2}}}{2\left\{\left(\frac{1}{R_{1}}+\frac{1}{R_{x}}+\frac{1}{R_{n}}\right) \frac{1}{R_{z}}+g_{m_{1}} g_{m_{2}}\right\}}, S_{R_{1}}^{Q_{0}}=\frac{\frac{1}{R_{1}}\left[-\frac{1}{2 R_{z}}(D)+\left\{\left(\frac{1}{R_{1}}+\frac{1}{R_{x}}+\frac{1}{R_{n}}\right) \frac{1}{R_{z}}+g_{m_{1}} g_{m_{2}}\right\}\left(C_{2}+C_{z}\right)\right]}{(D)\left\{\left(\frac{1}{R_{1}}+\frac{1}{R_{x}}+\frac{1}{R_{n}}\right) \frac{1}{R_{z}}+g_{m_{1}} g_{m_{2}}\right\}}
\end{aligned}
$$




$$
\begin{aligned}
S_{R_{x}}^{Q_{0}}= & \frac{1}{R_{x}}\left[-\frac{1}{2 R_{z}}(D)+\left\{\left(\frac{1}{R_{1}}+\frac{1}{R_{x}}+\frac{1}{R_{n}}\right) \frac{1}{R_{z}}+g_{m_{1}} g_{m_{2}}\right\}\left(C_{2}+C_{z}\right)\right] \\
(D)\left\{\left(\frac{1}{R_{1}}+\frac{1}{R_{x}}+\frac{1}{R_{n}}\right) \frac{1}{R_{z}}+g_{m_{1}} g_{m_{2}}\right\} & (D)\left\{\left(\frac{1}{R_{1}}+\frac{1}{R_{x}}+\frac{1}{R_{n}}\right) \frac{1}{R_{z}}+g_{m_{1}} g_{m_{2}}\right\} \\
S_{R_{n}}^{Q_{0}}= & \frac{\frac{1}{R_{n}}\left[-\frac{1}{2 R_{z}}(D)+\left\{\left(\frac{1}{R_{1}}+\frac{1}{R_{x}}+\frac{1}{R_{n}}\right) \frac{1}{R_{z}}+g_{m_{1}} g_{m_{2}}\right\}\left(C_{2}+C_{z}\right)\right]}{(D)\left\{\left(\frac{1}{R_{1}}+\frac{1}{R_{x}}+\frac{1}{R_{n}}\right) \frac{1}{R_{z}}+g_{m_{1}} g_{m_{2}}\right\}} \\
S_{R_{z}}^{Q_{0}}= & \frac{\frac{1}{R_{z}}\left[-\frac{1}{2}\left(\frac{1}{R_{1}}+\frac{1}{R_{x}}+\frac{1}{R_{n}}\right)(D)+\left\{\left(\frac{1}{R_{1}}+\frac{1}{R_{x}}+\frac{1}{R_{n}}\right) \frac{1}{R_{z}}+g_{m_{1}} g_{m_{2}}\right\}\left(C_{1}+C_{x}+C_{n}\right)\right]}{(D)(E)}, \\
S_{C_{1}}^{Q_{0}}= & \frac{C_{1}\left[\frac{1}{2}\left(C_{2}+C_{z}\right)(D)-(E) \frac{1}{R_{z}}\right]}{(D)(E)}, S_{C_{2}}^{Q_{0}}=\frac{C_{2}\left[\frac{1}{2}\left(C_{1}+C_{x}+C_{n}\right)(D)-(E)\left(\frac{1}{R_{1}}+\frac{1}{R_{x}}+\frac{1}{R_{n}}\right)\right]}{(D)(E)}, \\
S_{C_{z}}^{Q_{0}}= & \frac{C_{z}\left[\frac{1}{2}\left(C_{1}+C_{x}+C_{n}\right)(D)-(E)\left(\frac{1}{R_{1}}+\frac{1}{R_{x}}+\frac{1}{R_{n}}\right)\right]}{S_{C_{n}}^{Q_{0}}=}, S_{C_{x}}^{Q_{0}}=\frac{C_{x}\left[\frac{1}{2}\left(C_{2}+C_{z}\right)(D)-(E) \frac{1}{R_{z}}\right]}{C_{n}\left[\frac{1}{2}\left(C_{2}+C_{z}+C_{p}\right)(D)-(E) \frac{1}{R_{z}}\right]},
\end{aligned}
$$

where

$$
(D)=\left(\frac{C_{1}}{R_{z}}+\frac{C_{x}}{R_{z}}+\frac{C_{n}}{R_{z}}+\frac{C_{2}}{R_{1}}+\frac{C_{z}}{R_{1}}+\frac{C_{2}}{R_{x}}+\frac{C_{z}}{R_{x}}+\frac{C_{2}}{R_{n}}+\frac{C_{z}}{R_{n}}\right),(E)=\left(C_{1} C_{2}+C_{1} C_{z}+C_{2} C_{x}+C_{z} C_{x}+C_{2} C_{n}+C_{n} C_{z}\right) .
$$

From the above mentioned sensitivity values, it is easy are no more than half in magnitude. to figure out that all the active and passive sensitivities 